\title{
Muscle strength, endurance and recovery in the post-infection fatigue syndrome
}

\author{
A R LLOYD, J PHALES, S CGANDEVIA
}

From the Unit of Clinical Neurophysiology, Department of Neurology and the Departments of Immunology and Infectious Diseases, The Prince Henry Hospital and the School of Medicine, University of New South Wales, Sydney, Australia

SUMMARY A test of muscle strength and "fatiguability" was administered to 20 normal subjects and 20 patients suffering from post-infection fatigue syndrome. Maximal isometric torque for the elbow flexors was measured before, during and after an endurance sequence of 18 maximal static contractions (10 s duration, $10 \mathrm{~s}$ rest interval). The maximal isometric strength was not significantly different between the patient and control groups. The relative torque produced at the end of the series of 18 static contractions did not differ significantly between patients and normal subjects. In the patients with post-infection fatigue syndrome there was impairment of the recovery of peak torque at 10 minutes after the endurance sequence $(p<0.02)$. The prominent subjective complaint of muscle fatigue in patients with post-infection fatigue syndrome contrasts with the relatively normal behaviour of their muscles during a controlled test of fatigue. The syndrome may include a disordered perception of achieved force and exertion.

The syndrome of persistent fatigue after a viral or other infection occurs commonly, and has been variously designated as post-viral fatigue syndrome, ${ }^{1}$ myalgic encephalomyelitis, ${ }^{2}$ chronic Epstein-Barr virus infection ${ }^{3}$ and recently as post-infection fatigue syndrome (PIFS). ${ }^{4}$ Profound muscle fatigue, precipitated by minimal physical activity is the major complaint amongst patients with PIFS. Despite this prominent symptom, little objective abnormality has been found in the muscles of affected individuals. Muscle biopsies have demonstrated inconsistent and mild, non-specific changes on both light and electron microscopic examination. ${ }^{12}$ Standard electromyography, has similarly shown no definite abnormality. ${ }^{15}$ Single-fibre electromyography has been reported to demonstrate increased jitter, but without any impulse blocking. ${ }^{6}$ Increased jitter alone does not account for the symptom of muscle fatigue, as failed transmission in the motor unit evidenced by impulse blocking on the electromyograph is required to produce sub-maximal contraction of the muscle. An abnormal early intracellular acidosis has been

Address for reprint requests: Dr S C Gandevia, Unit of Clinical Neurophysiology, The Prince Henry Hospital, PO Box 233, Matraville, Sydney 2036, Australia.

Received 1 March 1988 and in revised form 10 May 1988. Accepted 13 May 1988 reported in the exercised muscle of patients with PIFS, ${ }^{7}$ based on ${ }^{31} \mathbf{P}$ nuclear magnetic resonance imaging. As the latter two studies did not include control subjects, the significance of their findings remains unclear. Biochemical analysis of a range of mitochondrial and glycolytic enzymes in muscles of patients with PIFS has shown no abnormality. ${ }^{8}$

To document objectively the muscle fatiguability in voluntary contractions, we measured maximal isometric strength before, during and after a series of maximal contractions of the elbow flexors. This technique has been used previously to study isometric strength and endurance in normal subjects and in various patient groups. ${ }^{9-12}$

\section{Patients and methods:}

Consecutive patients who fulfilled our diagnostic criteria for PIFS $^{4}$ (table 1), were subjects in this study. These criteria incorporate the characteristic features of the history (including subjective fatiguability of muscles), abnormalities on physical examination (including lymphadenopathy), and abnormal laboratory investigations (including $T$ cell lymphopenia and cutaneous anergy) in a weighted scale analogous to the Duckett-Jones criteria for the diagnosis of rheumatic fever. Twenty-five patients were tested, five of whom were subsequently excluded (see below). The patient group included 10 males and 10 females (table 2). Abnormal cell-mediated immunity was present in 15 of the 20 patients (T4 lymphopenia in nine patients, T8 lymphopenia in 11 
Table 1 Criteria for the diagnosis of PIFS

To fulfil the criteria a patient must have:

Chronic persisting or relapsing fatigue of a generalised nature, causing major disruption of usual daily activities, present for greater than six months.

plus

Two major criteria OR one major AND three minor criteria (below):

(1) Symptoms: Persistent at least six months continuously, or relapsing on three or more occasions with a similar pattern over six months or more.

Major Minor

Concentration/memory impairment Myalgia

Arthralgia

Headaches

Depression

Tinnitus

Paraesthesiae

(2) Signs: Present on at least one occasion subsequent to the initial illness.

$\begin{array}{ll}\text { Major } & \text { Minor } \\ \text { Lymphadenopathy } & \text { Pharyngitis } \\ \text { Localised muscle tenderness } & \end{array}$

Localised muscle tenderness

(3) Immunological assessment:

Major

T8 or T4 lymphopenia (absolute count)

Minor

Cutaneous anergy

OR Hypoergy

patients and cutaneous anergy in three). Two patients developed PIFS following serologically documented Epstein-Barr virus (EBV) infection, and another after serologically proven toxoplasmosis. Three patients had persistent (greater than 12 months) EBV IgG (VCA) titres > 640. One patient (above) had a persistent toxoplasma IgG titre (HA) $>512$. The patients had been suffering from PIFS for a mean of 34 months (range 10-120 months). None of the patients were seeking compensation for PIFS as a work-related illness.

Control subjects were selected from hospital employees who were unfamiliar with muscle strength testing. Twentysix control subjects were tested, and six were subsequently excluded (see below). The groups were matched for age and height (table 2). The male patients were heavier than their male control subjects (mean 76 vs. $65.6 \mathrm{~kg}$; p $<0.05$ ). No weight difference was apparent in the females (table 2). The general activity of all subjects during the preceding 2 months was assessed using an administered questionnaire, ${ }^{10}$ and the activity was then graded by a 5-tier scale that ranged from "sedentary" (score $=0$ ) to "endurance training for sporting events" (score = 4). Patients and control subjects were similarly matched for general activity ("training status", table 2). Informed consent was obtained from all subjects, and the study was approved by the appropriate institutional ethics committees.

Strength and endurance of the elbow flexors were examined because established techniques ${ }^{9-12}$ exist for assessment of this muscle group. The specific muscle group selected was thought not to be critical in view of the generalised nature of the muscle fatigue in patients with PIFS. ${ }^{1-4}$ Testing was performed with the subject seated and the right arm fixed to a vertical isometric myograph. The forearm was fully supinated, and the elbow was flexed at $90^{\circ}$. Torque (which is directly proportional to force) was measured with a bridgecircuit based on four strain gauges fixed to the base of the bar. The subject's maximal isometric strength was determined as the maximal torque in three brief ( $1-3 \mathrm{~s})$ contractions. There was minimal variation in the torque obtained in these trial maxima $( \pm 10 \%)$. Subjects were then asked to complete a series of 18 maximal isometric contractions, with a duty cycle of $50 \%$ (contractions of $10 \mathrm{~s}$ duration, separated by rest intervals of $10 \mathrm{~s}$ ). Brief static contractions were also recorded at $1 \mathrm{~min}, 5 \mathrm{~min}$, and $10 \mathrm{~min}$ after the completion of the "endurance sequence" of 18 sustained contractions. Subjects were given uniform instructions before the test, and then vigorously exhorted to perform to their maximum with standardised verbal encouragement throughout the procedure. A graphic display of the recorded torque provided continuous visual feedback to the subject, although the gain of this record was periodically altered to prevent assessment by the subject of the exact force achieved.

Data from the procedure were sampled at $50 \mathrm{~Hz}$ by computer and stored for subsequent analysis. The peak and average force maintained during each contraction were assessed. The measurement of average force was made by integration of the area under the curve of each contraction. Previous studies have demonstrated a high correlation between peak and average force measurements during repeated static efforts. ${ }^{910}$

The complete record of all force recordings was examined by an investigator, unaware of the clinical status of the subject. The data were classified as unsuitable for inclusion if: (1) the peak force was attained later than 3 seconds after the onset of the contraction, in two or more contractions; (2) a transient loss of force ( $50 \%$ or more of the peak force) occurred in two or more contractions; (3) a peak force in excess of the initial maximal force, occurred in a contraction after the third contraction in the endurance sequence. On the basis of these criteria, 11 subjects (five patients, six control subjects) were excluded.

In a second study, nine subjects (five controls, four patients) performed the complete testing sequence twice with

Table 2 Characteristics of subject groups. Ten subjects in each group. Values expressed as mean 1 SD (in parenthesis)

\begin{tabular}{llllll}
\hline & $\begin{array}{l}\text { Age } \\
(y r s)\end{array}$ & $\begin{array}{l}\text { Height } \\
(\mathrm{cm})\end{array}$ & $\begin{array}{l}\text { Weight } \\
(\mathrm{kg})\end{array}$ & $\begin{array}{l}\text { Training } \\
\text { Status** }\end{array}$ & $\begin{array}{l}\text { Strength } \\
(\text { Nm) }\end{array}$ \\
\hline Male controls & $35(11)$ & $176(7)$ & $65(7)$ & $1 \cdot 3(0 \cdot 5)$ & $66(10)$ \\
Male patients & $38(16)$ & $176(7)$ & $76(10)$ & $1 \cdot 3(0 \cdot 5)$ & $75(14)$ \\
Female controls & $35(12)$ & $163(6)$ & $60(8)$ & $1 \cdot 1(0 \cdot 3)$ & $44(6)$ \\
Female patients & $35(14)$ & $163(5)$ & $57(8)$ & $1 \cdot 1(0 \cdot 3)$ & $41(6)$ \\
\hline
\end{tabular}

*p 0.02

* p $1-5$ scale, see Methods 
a 3 hour rest interval between the tests. This study was designed to assess the commonly reported symptom of profound muscle fatigue occurring some hours after exercise. The rest interval of 3 hours was chosen as typical of the time of onset of this subjective fatigue in these subjects. It also allowed re-testing prior to the development of myalgia, which commonly occurs 12-24 hours after exercise in patients with PIFS. ${ }^{2}$ No subjects developed myalgia, nor was there demonstrable muscle tenderness at the time of the re-testing.

As reported in previous studies, ${ }^{9-12}$ the peak force achieved by the subjects fell steadily in successive contractions below that of the initial maximal voluntary contraction (MVC) (fig 1). The relative decline in the peak and mean force during the sequence of 18 sustained contractions was taken as an index of muscle endurance. In the subsequent three, brief maximal contractions (at 1, 5 and 10 $\mathrm{min}$ ), the force returned towards the initial MVC. This 10 minute period is defined as the recovery phase of the study. The term fatigue is used throughout the text to denote the failure of a muscle to exert the maximal voluntary torque without implying that the responsible mechanism is central or peripheral.

Differences between the subject groups in age, height, weight and training status were assessed using unpaired twotailed $t$ tests. The peak torque values, expressed as a percentage of the initial MVC, were used to compare data from the endurance sequence. The mean of the peak torque values obtained in the final three contractions of the endurance

sequence (that is, contractions $16,17,18$ ) was expressed as a percentage of the initial MVC. This percentage was designated as the "fatigue index". Evaluation of the data from the recovery contractions was also made by taking the mean of the peak force achieved in the final three contractions of the endurance sequence, and then expressing the forces during recovery as a percentage of this mean. The percentage obtained in the final recovery contraction (ie at $10 \mathrm{~min}$ ) was designated as the "recovery index". The data from these indices were analysed using an unpaired two-tailed $t$ test.

\section{Results}

The maximal isometric muscle strength of the male patients with PIFS (mean 74.8, SD $14.4 \mathrm{Nm}$ ), was greater than that of the control subjects $(65 \cdot 7, \mathrm{SD} 10 \cdot 1$ $\mathrm{Nm}$; table 2), but not significantly so. The trend in favour of the patients in this strength measurement may be due to the greater weight of the patients (76, SD $10 \mathrm{~kg}$ vs 65 , SD $7 \mathrm{~kg}$ ). The maximal isometric strength of the female patients was $41 \cdot 4$, SD $5.7 \mathrm{Nm}$, and of the female control subjects was $43 \cdot 7$, SD $6 \cdot 4$ $\mathrm{Nm}$. These values were not significantly different.

During the series of maximal isometric contractions, peak force fell in all subjects to approximately $65 \%$ (range: $45-81 \%$ ). This progressive decline in the force achieved in the repetitive con-

\section{Endurance}

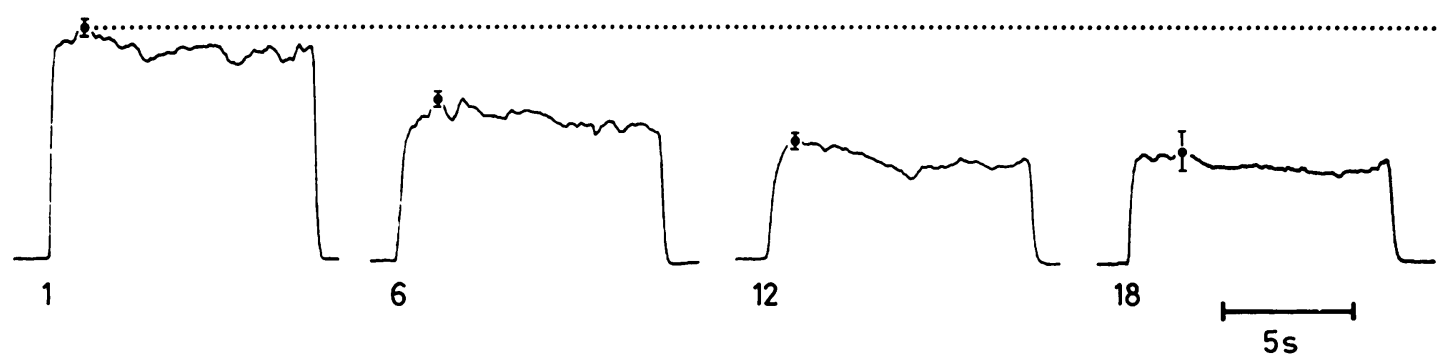

Recovery

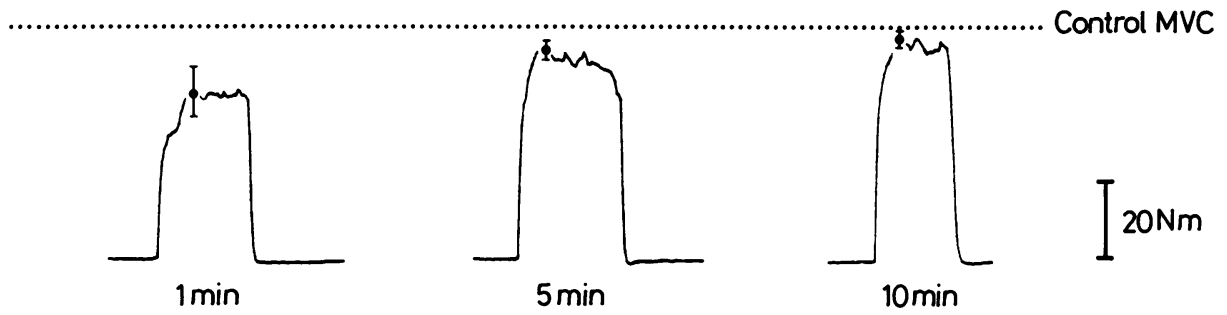

Fig 1 Records from a typical experiment in a control subject. Four contractions (1st, 6th, 12th, 18th) from the endurance sequence are shown (upper panel). The three recovery contractions are shown in the lower panel (1 min, $5 \mathrm{~min}, 10 \mathrm{~min}$ ). The peak torque obtained in the same contractions in repeated testing sessions are demonstrated by the standard deviation bars. 
tractions over the 6 minutes of the endurance phase (figs 2 and 3) is similar to that reported in previous studies. ${ }^{9-11}$ There was no significant difference in the decline in peak force, as measured by the "fatigue index", between the male and female subjects, and no significant difference in the "fatigue index" when the 20 patients were compared with the 20 control subjects $(63 \cdot 0$, SD $7 \cdot 3$ vs $66 \cdot 6$, SD $9 \cdot 4 \%)$. When the male and female subjects were analysed separately (figs 2 and 3), the female patients showed significantly more fatigue than the control subjects $(63 \cdot 0$, SD 6.5 vs $71 \cdot 3$, SD $9.6 \% ; p<0.05)$. There was an opposite trend in the male patients but this was not significant. Two male patients had peak torque values in the endurance sequence which fell repeatedly above the $95 \%$ confidence limits for the equivalent contractions in the male control subjects. Two female patients had peak torque values in the endurance sequence which fell repeatedly below the $95 \%$ confidence limits for the equivalent contractions in the female control subjects. The values for these four patients were the reason for the small group differences (above).

In the nine subjects (five controls, four patients) who repeated the test on one or more separate occasions, both the maximal torque and the pattern of fatigue and recovery were reproducible (fig 1 ; see also $\mathrm{refs}^{910}{ }^{13}$ ). The maximal torque, the "fatigue index" and the "recovery index" all usually differed by less than $10 \%$ in comparison with the respective values in previous testing.

In the recovery phase, only the peak torque mea- surements are relevant, because the contractions were brief, unsustained efforts designed to achieve maximal force but not to induce fatigue (see Patients and Methods). Given that there was no significant difference between the male and female subjects during the endurance sequence, data from the recovery phase testing of both sexes were analysed together. In the initial recovery contraction (at $1 \mathrm{~min}$ ), the patients achieved a peak torque of 110 , SD $12 \%$ of the average of the peak torque achieved in the final three contractions of the endurance sequence (contractions $16,17,18)$. At this stage (1 min), the control subjects achieved 115 , SD $12 \%$. In the second recovery contraction (at $5 \mathrm{~min}$ ), the patients achieved 117 , SD $13 \%$ in comparison with 123 , SD $18 \%$ in the control subjects. In the final recovery contraction (the "recovery index"), the patients recovered less than the control subjects (118, SD 14 vs 131 , SD $19 \%$; p < 0.02). Separate analysis of the data for the sexes showed this difference to be significant for the males but not the females.

When the individual patient records were examined it was apparent that there was no exceedingly good recovery of peak torque in an individual control subject to bias the control data in favour of recovery. Three male patients had peak torque values in the recovery sequence which fell outside the $95 \%$ confidence limits for the equivalent contractions in the male control data. The "recovery index" for these three patients was greater than three standard deviations below the mean of the index in the male control

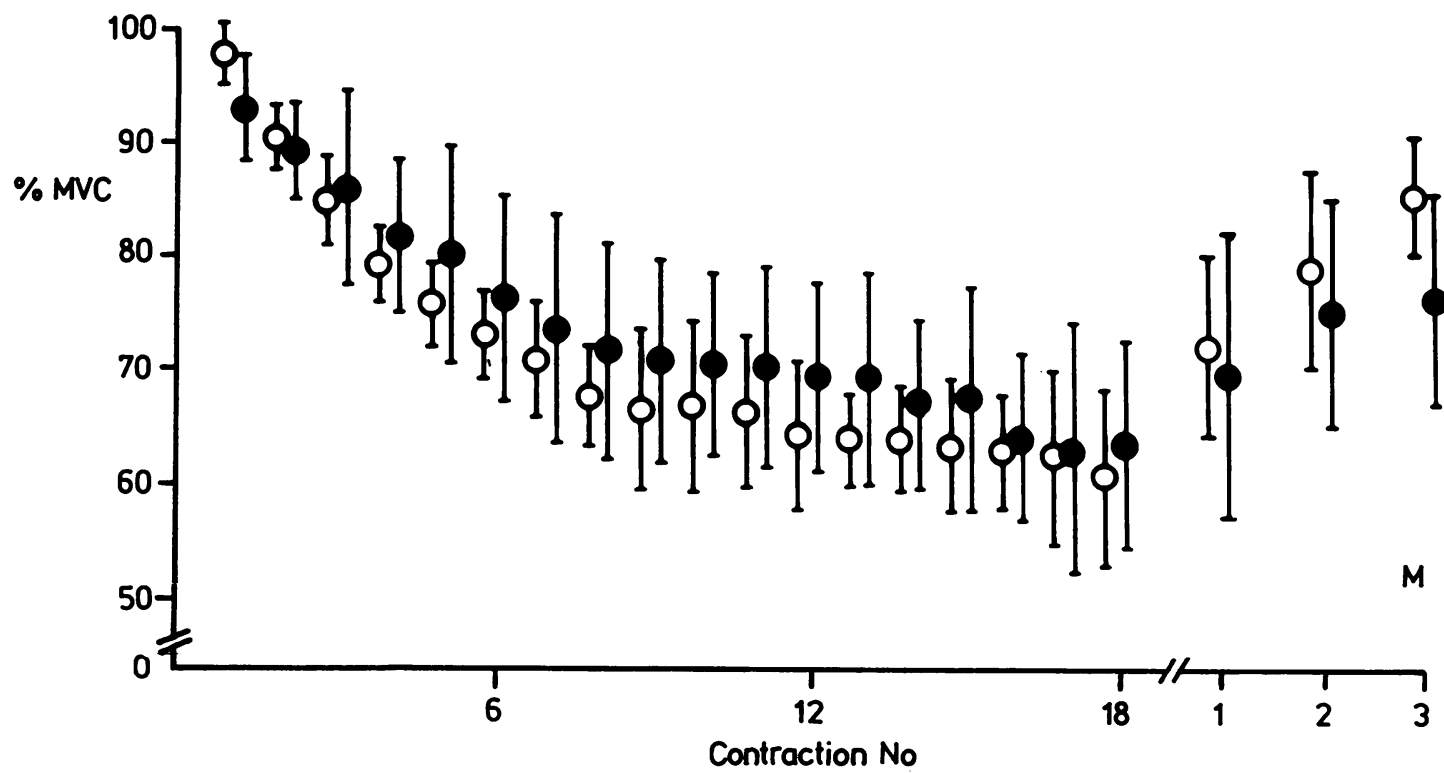

Fig 2 Maximal performance of the elbow flexors tested with 18 maximal static contractions (10 $s$ duration, $10 \mathrm{~s}$ rest interval: $50 \%$ duty cycle), and three brief maximal contractions in the recovery phase in 10 male patients with PIFS (closed circles), and 10 male control subjects. Each data point represents the mean and SD for the peak torque attained in each contraction expressed as a percentage of the peak torque of the initial maximal voluntary contractions (MVC). 


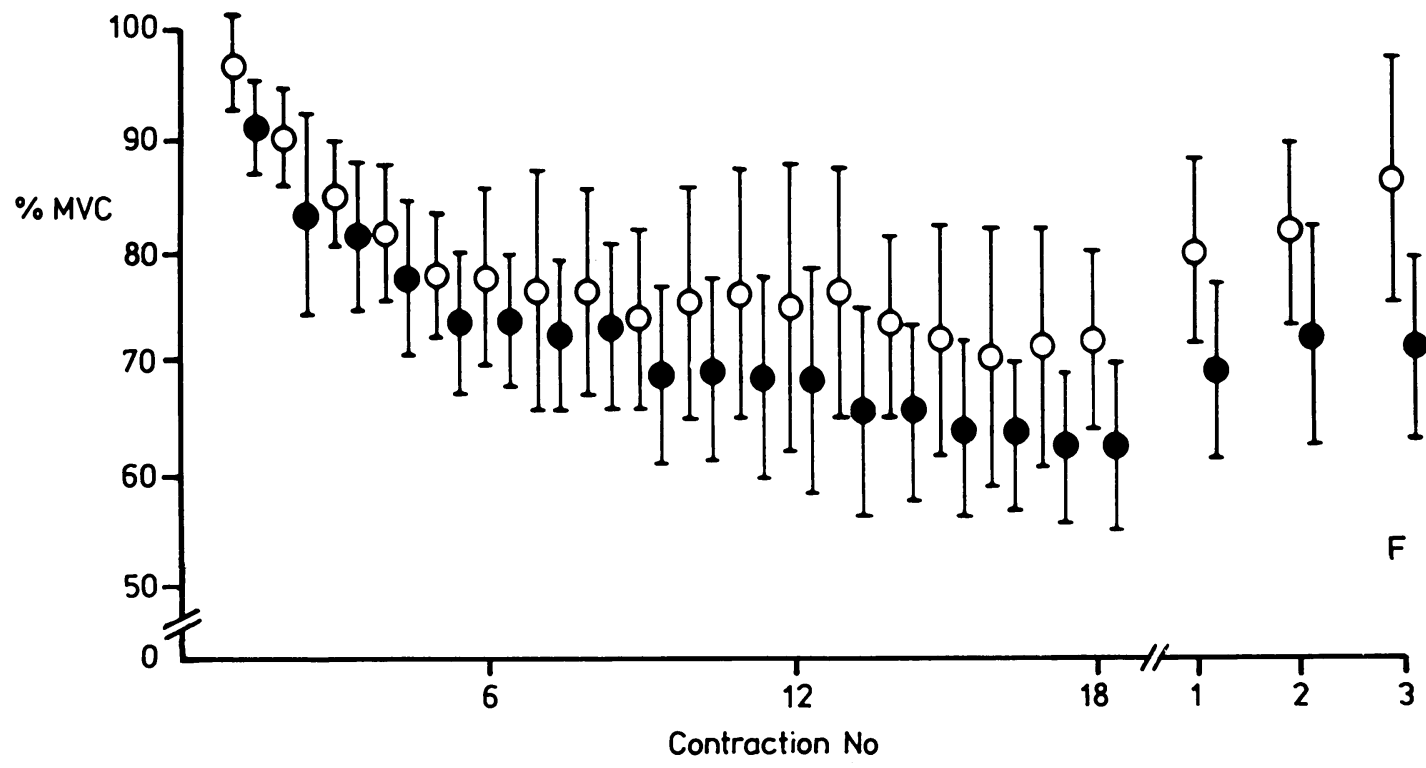

Fig 3 Maximal performance of the elbow flexors tested with 18 maximal static contractions (10 s duration, $10 \mathrm{~s}$ rest interval: $50 \%$ duty cycle), and three brief maximal contractions in the recovery phase in 10 female patients with PIFS (closed circles) and 10 female control subjects (open circles). Each data point represents the mean and SD for the peak torque attained in each contraction expressed as a percentage of the peak torque of the initial maximal voluntary contractions (MVC).

subjects. On the other hand the peak torque values in the recovery sequence of all of the female patients fell within 2.5 standard deviations of the equivalent contractions in the female control subjects.

When the full muscle test was repeated after a 3 hour rest, the initial maximal isometric strength achieved in the second session was approximately $90 \%$ (mean $88.9 \%$; range $79-102 \%$ ) of that of the earlier study in both patients and control subjects. For both the patients and normal subjects, the "fatigue index" and the "recovery index" in the second session were not significantly different from those in the first

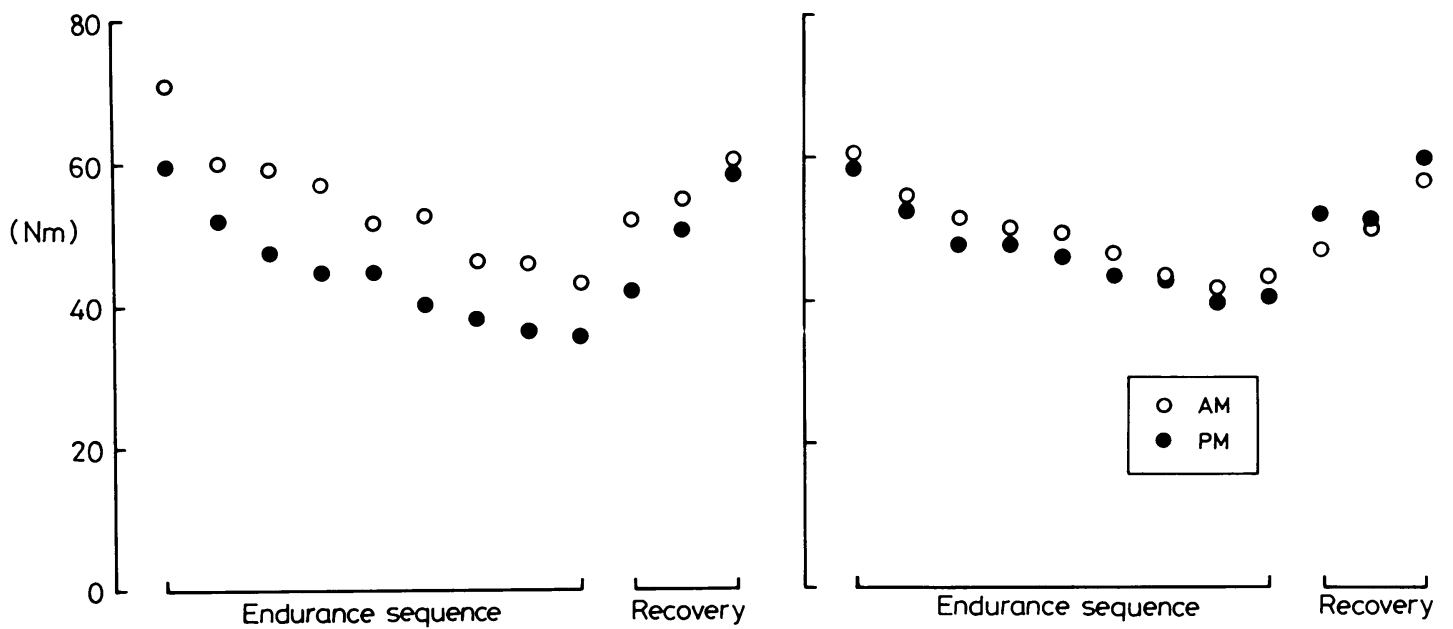

Fig 4 Endurance and recovery sequences in the testing session repeated after a 3 hour interval in a male control subject (left), and a male patient (right). The absolute torque values are shown from the first testing session (open circles, labelled 'AM' for the morning session) and from the second testing session (closed circles, labelled 'PM' for the afternoon session). The mean of consecutive pairs of contractions in the endurance sequence of 18 contractions are shown, as are each of the contractions in the recovery phase ( $1 \mathrm{~min}, 5 \mathrm{~min}$ and $10 \mathrm{~min}$ ). 
session. A set of typical data from a patient and control subject is shown in fig 4 .

\section{Discussion}

This study documents normal maximal isometric strength in patients with PIFS. Thus there is no objective muscle weakness in patients with this syndrome. This finding is consistent with the characteristically normal neurological examination in subjects with PIFS. ${ }^{124}$ It contrasts with the reported finding of reduced isometric strength in patients with acute infectious diseases (predominantly viral infections); ${ }^{14}$ although in this previous study, myalgia may have limited the performance of a significant proportion of the patients.

The results of this study highlight a discrepancy between the prominent complaint of fatigue and exercise intolerance reported by patients with PIFS, and the lack of a comparable abnormality in the assessment of muscle endurance. The failure to demonstrate any marked abnormality in the pattern of endurance in the muscles of the patients is consistent with the lack of major abnormalities reported in microscopic ${ }^{1}$, electrophysiological ${ }^{15}$ and biochemical ${ }^{8}$ studies of muscles of patients with PIFS.

A significant impairment of recovery of maximal isometric strength after the endurance sequence testing was demonstrated in the patients in comparison with the control subjects. In the majority of the patients, this deficit failed to disturb the muscle performance over the preceding $360 \mathrm{~s}$ of maximal voluntary force production. Two female patients only, demonstrated significantly reduced strength in the endurance sequence. Three male patients only, had reduced maximal isometric strength in the recovery sequence testing. By far the majority of the patients, all of whom fulfilled the diagnostic criteria for PIFS and all of whom complained of profound muscle fatigue demonstrated normal maximal isometric strength, endurance and recovery in this study.

An important consideration in any test of maximal voluntary strength is the degree to which the subjects recruit motoneurons of the relevant muscles. There is considerable evidence that well motivated subjects, provided that they are free of muscle, joint or other pain, ${ }^{15}$ are capable of sustaining voluntarily, the maximal force possible from a muscle group (that is, show no "central fatigue"). This is based upon the failure of interpolated electrical stimuli to the nerve or muscle to increase the force output from the voluntarily contracting muscle ${ }^{16-18}$ (for review see ref 19 ). The ability to activate a muscle maximally by voluntary effort has been documented for limb and even respiratory muscles, ${ }^{20}$ and has recently been shown for the elbow flexors. ${ }^{21}$ In view of these findings and the surprising similarities in maximal strength and endurance between the control and patient groups in this study, it is unlikely that poor motivation played any role during the endurance sequences studied here. Nevertheless, it is possible that the failure of the patients with PIFS to recover as quickly as normal could result from inability to generate a maximal motor command from the central nervous system.

The repeated maximal isometric contractions produced by the subjects in this study are clearly greater than the relatively milder demands placed on the muscles by the patients in their normal activities of daily life. However, such normal activities do in fact produce the complaint of profound muscle fatigue in these patients as the predominant feature of the syndrome. Therefore the demonstration of normal muscle function in the majority of patients using this vigorous testing regimen, implies that the muscle is unlikely to be the major site of dysfunction in patients with PIFS.

The immunological and serological abnormalities noted in the patients in this study are prevalent in patients with PIFS. ${ }^{134}$ These abnormalities have been linked in a hypothesis suggesting low-grade persistent intracellular infection and localised lymphokine (interferon) release producing a generalised disorder of cell membranes (including within the CNS) as the pathophysiological basis of PIFS. ${ }^{22}$ Fatigue and other symptomatology suggestive of PIFS have been reported as common complaints in patients receiving therapy with lymphokines such as recombinant alpha interferon. ${ }^{23}$

Given the normal strength, endurance and recovery of the majority of patients with PIFS as documented in this study, it is possible that the "fatigue" of PIFS is associated with an abnormality of perception of muscle force and effort rather than of actual force production.

This work was supported by the National Health and Medical Research Council of Australia. We are grateful to Drs D Burke, D Gillies and D Wakefield for comments on the manuscript.

\section{References}

1 Behan PO, Behan WMH, Bell EJ. The postviral fatigue syndrome: an analysis of the findings in 50 cases. $J$ Infect 1985;10:211-22.

2 Proceedings of the Symposium of the Council of the Royal Society of Medicine on 'Epidemic myalgic encephalomyelitis'. Postgrad Med J 1978;54:709-77.

3 Straus SE, Tosato G, Armstrong G. Persisting illness and fatigue in adults with evidence of Epstein-Barr virus infection. Ann Intern Med 1985;102:7-18.

4 Lloyd A, Wakefield D, Boughton C, Dwyer J. What is myalgic encephalomyelitis? Lancet 1988;i:1286-7.

5 Richardson AT. Electromyographic studies of patients with 'epi- 
demic neuromyasthenia' at the Royal Free Hospital. Postgrad Med J 1978;54:745.

6 Jamal GA, Hansen S. Electrophysiological studies in postviral fatigue syndrome. J Neurol Neurosurg Psychiatry 1985; 48:691-94.

7 Arnold DI, Bore PJ, Radda GK, Styles P, Taylor DJ. Excessive intracellular acidosis of skeletal muscle on exercise in a patient with a post viral fatigue syndrome. Lancet 1984;i:1367-69.

8 Byrne E, Trounce I. Chronic fatigue and myalgia syndrome: mitochondrial and glycolytic studies in skeletal muscle. $J$ Neurol Neurosurg Psychiatry 1987;50:743-46.

9 Gandevia SC, McKenzie DK, Neering IR. Endurance properties of respiratory and limb muscles. Resp Physiol 1983;53:47-61.

10 McKenzie DK, Gandevia SC. Strength and endurance of inspiratory, expiratory and limb muscles in asthma. Am Rev Resp Dis 1986;134:999-1004.

11 McKenzie DK, Gandevia SC. Influence of muscle length on human inspiratory and limb muscle performance. Resp Physiol 1987;67:171-82.

12 Colebatch JG, Gandevia SC, Spira PJ. Voluntary muscle strength in hemiparesis: distribution of weakness at the elbow. $J$ Neurol Neurosurg Psychiatry 1986;49:1019-24.

13 Viitasalo JT, Saukkonen S, Komi PV. Reproducibility of measurements of selected neuromuscular performance variables in man. Electromyogr Clin Neurophysiol 1980;20:487-501.

14 Friman G. Effect of acute infectious disease on isometric muscle strength. Scand J Clin Lab Invest 1977;37:303-8.

15 Rutherford OM, Jones DA, Newham DJ. Clinical and experimental application of the percutaneous twitch superimposition technique for the study of human muscle activation. J Neurol Neurosurg Psychiatry 1986;49:1288-91.

16 Merton PA. Voluntary strength and fatigue. J Physiol (Lond) 1954;123:553-64.

17 Bigland B, Lippold OC. Motor unit activity in the voluntary contraction of human muscle. J Physiol (Lond) 1954;125:322-35.

18 Belanger AY, McComas AJ. Extent of motor unit activation during effort. J Appl Physiol 1981;51:1131-35.

19 Bigland-Ritchie B, Woods JJ. Changes in muscle properties and neural control during human muscular fatigue. Muscle Nerve 1984;7:691-9.

20 Gandevia SC, McKenzie DK. Activation of the human diaphragm during maximal static efforts. $J$ Physiol (Lond) 1985;367:45-56.

21 Gandevia SC, McKenzie DK. Maximal voluntary activation of human muscles at short muscle lengths. J Physiol (Lond) 1988 (In press).

22 Wakefield D, Lloyd AR. Pathophysiology of myalgic encephalitis. Lancet 1987;ii:918-9.

23 McDonald EM, Mann AH, Thomas HC. Interferons as mediators of psychiatric morbidity. An investigation in a trial of recombinant alpha interferon in hepatitis B carriers. Lancet 1987;ii:1175-9. 